\title{
Two-Lane Traffic Flow Simulation Model via Cellular Automaton
}

\author{
Kamini Rawat, Vinod Kumar Katiyar, and Pratibha Gupta \\ Department of Mathematics, IIT Roorkee, Roorkee 247667, India \\ Correspondence should be addressed to Kamini Rawat, rawatkamini@gmail.com
}

Received 18 February 2011; Accepted 16 June 2011

Academic Editor: Nandana Rajatheva

Copyright ( 2012 Kamini Rawat et al. This is an open access article distributed under the Creative Commons Attribution License, which permits unrestricted use, distribution, and reproduction in any medium, provided the original work is properly cited.

\begin{abstract}
Road traffic microsimulations based on the individual motion of all the involved vehicles are now recognized as an important tool to describe, understand, and manage road traffic. Cellular automata (CA) are very efficient way to implement vehicle motion. $\mathrm{CA}$ is a methodology that uses a discrete space to represent the state of each element of a domain, and this state can be changed according to a transition rule. The well-known cellular automaton Nasch model with modified cell size and variable acceleration rate is extended to two-lane cellular automaton model for traffic flow. A set of state rules is applied to provide lane-changing maneuvers. S-t-s rule given in the BJH model which describes the behavior of jammed vehicle is implemented in the present model and effect of variability in traffic flow on lane-changing behavior is studied. Flow rate between the single-lane road and two-lane road where vehicles change the lane in order to avoid the collision is also compared under the influence of s-t-s rule and braking rule. Using results of numerical simulations, we analyzed the fundamental diagram of traffic flow and show that s-t-s probability has more effect than braking probability on lane-changing maneuver.
\end{abstract}

\section{Introduction}

Cellular automata (CA) is a mathematical machine that arises from very basic mathematical principles. Though they are remarkably simple at the start, CA has variety of applications. It has been used extensively for modeling of single-lane traffic. Some modifications are required to extend these models to two-lane traffic as these generally fail to explain the lane-changing behavior. First traffic flow model using the concept of one-dimensional CA was given by Nagel-Schrekenberg popularly known as Nasch model [1]. The simple mean-field theory approach assumes that the two neighboring sites are completely uncorrelated. A simple model for two-lane traffic was also investigated, but the update rules were not defined in the same manner as in Nasch model. It was found that the fundamental diagram for each lane is asymmetric but the maximum is shifted towards large values of vehicular density $\rho\left(\rho_{\max }>1 / 2\right)$. The twolane cellular automaton model based upon the single-lane CA introduced by Rickert et al. was examined [2]. It was concluded that for both symmetric and asymmetric version, maximum flow $q_{\max }$ is higher than twice the maximum flow of single-lane model. An introduction of small number of slow vehicles can initiate formation of clusters at smaller densities [3]. For asymmetric lane-changing rules, slow vehicles influence the system performance less than in symmetric case. In two-lane traffic flow model, if the car density is set within a range, the self-organization of the slow and fast lanes was observed in spite of symmetry between two lanes [4]. Several branches and hysteresis in flow-density graph are observed. Results relative to a simple CA model without periodic boundary condition for a highway with variable number of on-ramps were presented [5]. A 2D extended version of the 1D Fukui-Ishibashi model, elaborated by Wang et al. [6], was presented for single-lane traffic to take into account the exchange of vehicles between the first and the second lane [6]. In general lane-changing rule can be symmetric or asymmetric with respect to the lanes or to the vehicles. While symmetric rules treat both lanes equally, asymmetric rule sets especially have to be applied for the simulation of German highways, where lane changes are dominated by right lane preferences and a right lane overtaking ban [7]. A new CA model by introducing the Honk effect into the basic symmetric two-lane CA model was proposed in [8]. The set of lane-changing rules (STCA) suggested by Chowdhury et al. [9] was revised to take the Honk effect into account (H-STCA). Lane-changing frequency for fast 
and slow vehicles for both models, STCA and H-STCA, was compared, and it was found that introduction of the Honk enhances the performance of the mixed vehicle traffic in the intermediate density range. A simple lattice-based exclusion model which can be considered as a crude representation of traffic on a two-lane motorway was introduced [10]. The model was two-lane generalization of the asymmetric simple exclusion process which is known to reproduce some of the features of the single-lane traffic such as shocks and jams. Effect of an aggressive lane-changing behavior on a two-lane road in presence of slow vehicles and fast vehicles has been further studied [11]. Simulation results show that aggressive lane-changing behavior of fast vehicles can depress the plug formed by slow vehicles and improve traffic flow in mixed traffic in intermediate density region. A highway traffic flow model with blockage induced by an accident vehicle was introduced in which both symmetric and asymmetric lane-changing rules were adopted [12]. It is concluded by numerical simulation that accident vehicle not only causes a local jam behind it, but also causes vehicles to cluster in the bypass lane. Further it is found that vehicles will change lane more frequently when the traffic is inhomogeneous with an accident car. In presence of a signalized intersection, existence of a certain combination of density $\rho$ and cycle time which optimizes the traffic efficiency in a two-lane model due to overtaking is investigated [13].

In the present study the cell size is reduced and variable acceleration rate (rather than 1) is taken into account [14]. Here we consider two-lane traffic and adopt the symmetric lane-changing rules, which take into account the incentive and safety criteria. A slow-to-start (s-t-s) rule used in the widely known Benjamin-Johnson-Hui (BJH) CA model for single lane traffic simulation [15] is implemented to two-lane traffic simulation. BJH model is also discussed, and a slightly modified deceleration rule in order to simulate braking behaviors of vehicles more correctly is taken into account [2]. The slow-to-start rule in BJH model is applicable only to stationary vehicles, that is, vehicles that are completely blocked by the leading vehicle in the previous time step. This rule is not applicable to those vehicles which are stopped due to randomization in the previous time step. We choose $\mathrm{BJH}$ model in the present study for the reason that it has extremely simple transition rules which are easy to implement on 2lane road. We investigate the effect of s-t-s and braking rules over lane-changing maneuver among vehicles in twolane road and a detailed comparison of effect of braking probability and s-t-s probability over two-lane traffic flow is carried out using simulation. Simulation results show that variability in traffic flow has significant effect on lanechanging behavior of vehicle.

\section{Cellular Automaton Model}

The Nagel-Schrekenberg model is a probabilistic CA model for the description of single-lane highway traffic. Mathematically it is expressed by four rules given as follows.

Rule 1. Acceleration: $v_{i}^{(t+\delta t / 3)}=\min \left\{v_{i}^{(t)}+1, V_{\max }\right\}$.
Rule 2. Deceleration: $v_{i}^{(t+2 \delta t / 3)}=\min \left\{v_{i}^{(t+\delta t / 3)}, x_{i+1}^{t}-x_{i}^{t}-1\right\}$. Rule 3. Randomization: $v_{i}^{(t+\delta t)}=\max \left\{v_{i}^{(\mathrm{t}+2 \delta t / 3)}-1,0\right\}$.

Rule 4. Movement: $x_{i}^{(t+\delta t)}=x_{i}^{t}+v_{i}^{(t+\delta t)}$.

Where $x_{i}^{t}$ and $v_{i}^{t}$ are the position and speed of $i$ th vehicle at a time $t$ and $x_{i+1}^{t}-x_{i}^{t}-1$, the number of empty cells in front of $i$ th vehicle at time $t$, is called distance headway. A time step of $\delta t=1 \mathrm{sec}$, and the typical reaction time of driver with a maximum speed $V_{\max }=5$ cells $/$ time step, that is, $135 \mathrm{~km} / \mathrm{h}$ is taken in this model. Cell size is taken as 7.5 meters and maximum speed $V_{\max }=5$ cells/time step which indicates the minimum headway of two moving vehicles, and time interval is one second. This indicates that changing speed will only be $7.5 \mathrm{~m} / \mathrm{sec}, 15 \mathrm{~m} / \mathrm{sec}$, and $22.5 \mathrm{~m} / \mathrm{sec}$, and so on. To overcome this problem different cell sizes are modeled by recording observation from real word, and a reduced cell size of 0.5 meters and variable acceleration rate that depends upon the speed of particular vehicle, are taken into account. Under this fine discretization we can describe the vehicle moving process more properly. A light vehicle occupies 12 cells with $V_{\max }=60$ cells which correspond to $108 \mathrm{~km} / \mathrm{h}$ whereas heavy vehicle occupies 20 cells with $V_{\max }=40$ cells which correspond to $72 \mathrm{~km} / \mathrm{h}$. Rule 1 of Nasch model is modified as follows.

Rule 1. Acceleration: $v_{i}^{(t+\delta t / 3)}=\min \left\{v_{i}^{(t)}+a, V_{\max }\right\}$.

Where acceleration $a$ is determined as follows:

$$
a= \begin{cases}4, & \text { if } v_{n} \leq 12, \\ 3, & \text { if } 12<v_{n} \leq 22 \\ 2, & \text { if } v_{n}>22\end{cases}
$$

2.1. Two-Lane Cellular Automata Traffic Model. Most of the major roads are two-lane one-way roads. We consider a two-lane model with periodic boundary conditions, where additional rules defining the exchange of vehicles between the lanes are applied. For our model we adapt the Nasch model to provide vehicle movements. Any vehicle may perform lane-changing maneuver based on three criteria: incentive criterion, improvement criteria, and safety criteria [11].

Incentive criteria:

$$
d_{i,}<\min \left(v_{i}+a, V_{\max }\right) .
$$

Improvement criteria:

$$
d_{i, \text { other }}>d_{i}
$$

Safety criteria:

$$
d_{i, \text { back }}>V_{\max },
$$

where $d_{i \text {,other }}, d_{i \text {,back }}$ denote the number of empty cells between the $i$ th vehicle and its two neighbor vehicles in the other lane at time $t$, respectively. 


\section{Slow-to-Start Rule}

We now implement a further rule to two-lane model, which is referred to as slow-to-start rule [16]. This s-t-s rule is given by Benjamin-Johnson-Hui and popularly known as BJH CA model. S-t-s rule is applicable only to static vehicles. Mathematically this rule can be defined as

$$
\begin{gathered}
\text { If } v_{i}^{(t)}=0, \quad x_{i+1}^{t-1}-x_{i}^{t-1}-1=0, \\
v_{i}^{(t+\delta t / 3)}=v_{i}^{(t+2 \delta t / 3)}=v_{i}^{(t+\delta t)}=0,
\end{gathered}
$$

where $v_{i}^{t}$ is the speed of $i$ th vehicle at time $t$ and $\delta t$ is the time interval. The above-mentioned s-t-s rule is applied only to the stopped vehicles having 0 headway in the previous time step with s-t-s probability $q$. It implies that s-t-s rule has no effect on the vehicles stopped due to randomization in the previous time step.

\section{New Stochastic CA Model for Two Lanes}

CA model developed so far with modified cell size and variable acceleration rate and with implementation of slowto-start rule for two-lane traffic is given as

Rule 1.

$$
\begin{gathered}
v_{i}^{(t)}=0, \quad x_{i+1}^{t-1}-x_{i}^{t-1}-12=0 \\
v_{i}^{(t+\delta t / 3)}=v_{i}^{(t+2 \delta t / 3)}=v_{i}^{(t+\delta t)}=0 \quad \text { with s-t-s probability } q .
\end{gathered}
$$

Rule 2.

$$
v_{i}^{(t+\delta t / 3)}=\min \left\{v_{i}^{(t)}+a, V_{\max }\right\}
$$

Rule 3.

$$
v_{i}^{(t+2 \delta t / 3)}=\min \left\{v_{i}^{(t+\delta t / 3)}, x_{i+1}^{t}-x_{i}^{t}-12\right\} .
$$

Rule 4.

$v_{i}^{(t+\delta t)}=\max \left\{v_{i}^{(t+2 \delta t / 3)}-1,0\right\}$ with braking probability $p$.

Rule 5.

$$
x_{i}^{(t+\delta t)}=x_{i}^{t}+v_{i}^{(t+\delta t)} .
$$

Together with lane-changing rules:

$$
x_{i+1}^{t}-x_{i}^{t}-12<\min \left(v_{i}^{t+\delta t / 3}, V_{\max }\right)
$$

$d_{i, \text { other }}>d_{i}$ and $d_{i \text {,back }}>V_{\max }$ with lane changing probability $s$.

\section{Numerical Simulation}

The numerical simulation is carried out with randomly generated initial configurations on a closed track containing
10,000 cells which represents a simulated road section of $5 \mathrm{~km}$. The periodic boundary condition is that $\mathrm{N}$ vehicles were randomly distributed on both lanes. For each initial configuration of vehicles, results are obtained by averaging over 3600 time steps. For each density $\rho$, results are averaged over 10 different initial configurations.

The computational formulas used in numerical simulation are given as follows:

$$
\begin{aligned}
& \bar{\rho}_{i}=\frac{1}{T} \sum_{t=1}^{t=T} n_{i}(t), \\
& \bar{q}_{i}=\frac{1}{T} \sum_{t=1}^{t=T} m_{i}(t),
\end{aligned}
$$

where (13) represents the density of the vehicles on the $i$ th site over a time period $T . n_{i}(t)=0$ if $i$ th site is empty and $n_{i}(t)=1$ if $i$ th site is occupied by a vehicle at time $t$. Equation (14) represents flow of vehicles on ith site; $m_{i}(t)=1$, if at time $t-1$, there was a vehicle behind or at $i$ th site, and at time $t$, it is found after $i$ th site (i.e., a vehicle is detected passing by $i$ th site). Density and flow are measured and averaged out over a time period of $T$.

\section{Lane-Changing Behavior}

The behavior of lane-changing criteria can be explained if two criteria are fulfilled to initiate lane change. First, the situation on the other lane must be more convenient and second the safety rules must be followed. A probability $(1-s)$ is prescribed for vehicle to stay in the current lane (left lane). There are two other parameters: braking probability and slow-to-start probability. We investigate the effect of two parameters $p$ and $q$ on lane-changing behavior of vehicles. It can be shown by simulation that initially vehicles change lanes frequently and the lane changing rate drops rapidly as time evolves. Figures 1(a) and 1(b) show independent effects of s-t-s probability and braking probability when acting alone, respectively, over the lane-changing behavior of a periodic two-lane system, whereas Figure 1(c) shows the combined effect of two probabilities. With smaller values of $p$ and $q$, vehicles rarely change lanes. With increase in the value of parameters $p$ and $q$, the lane-changing tendency among the vehicle increases. This is due to the cluster formation at different locations on both lanes. With higher values of $p$ and $q$, there will always be cluster formation and between the two clusters there is sufficient space on the right lane for vehicles to change the lane, and lane change becomes more likely. Therefore maximum lane changes occur even at higher values of traffic density $\left(\rho>\rho_{\mathrm{c}}\right)$. Here we choose the lane-changing probability $s=0.8$, and it remains constant throughout the simulation. From Figures 1(a) and 1(b), it can be observed that an introduction of nonzero $q$ has a stronger influence than nonzero $p$ on lane-changing rate of vehicles in two-lane road. As described in BJH model, an introduction of nonzero s-t-s probability makes the queues less fragmented and the interqueue regions widen, as a result vehicles find enough gaps in the target lane to change the 


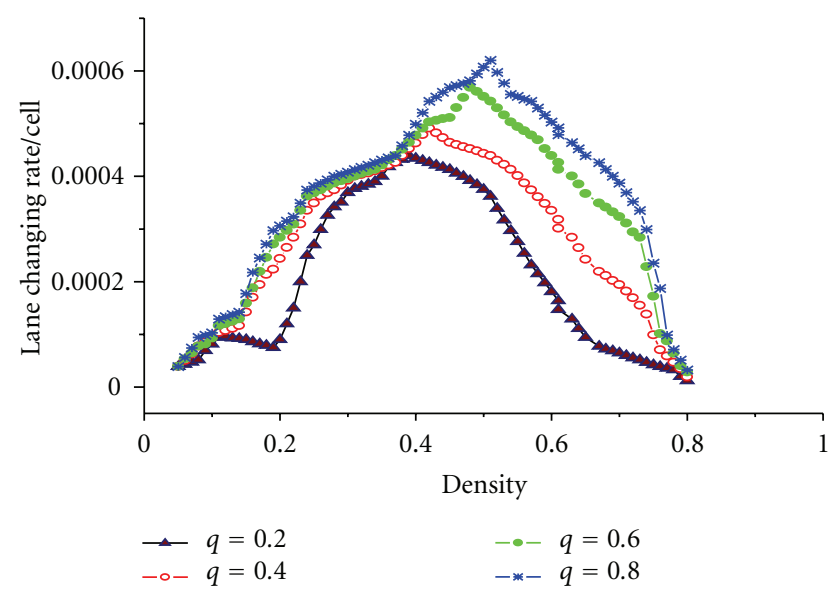

(a)

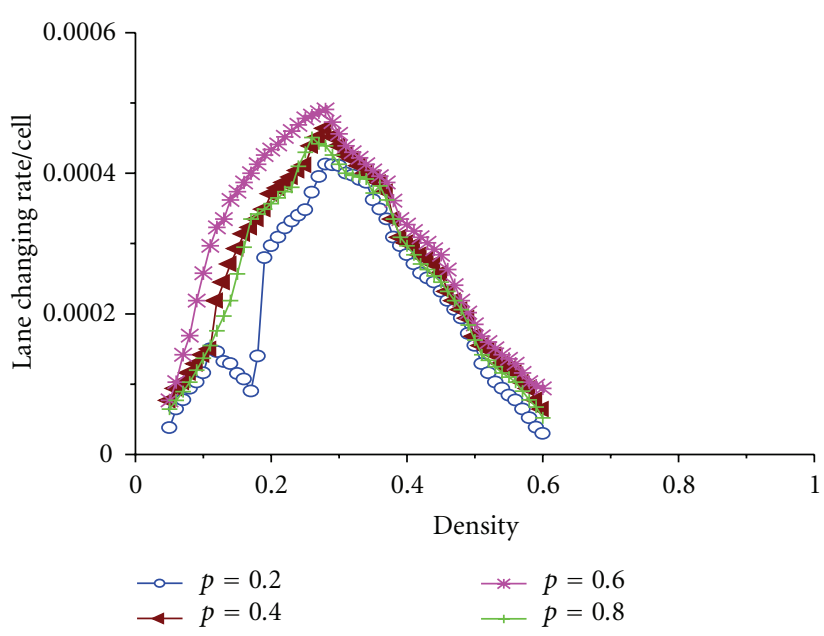

(b)

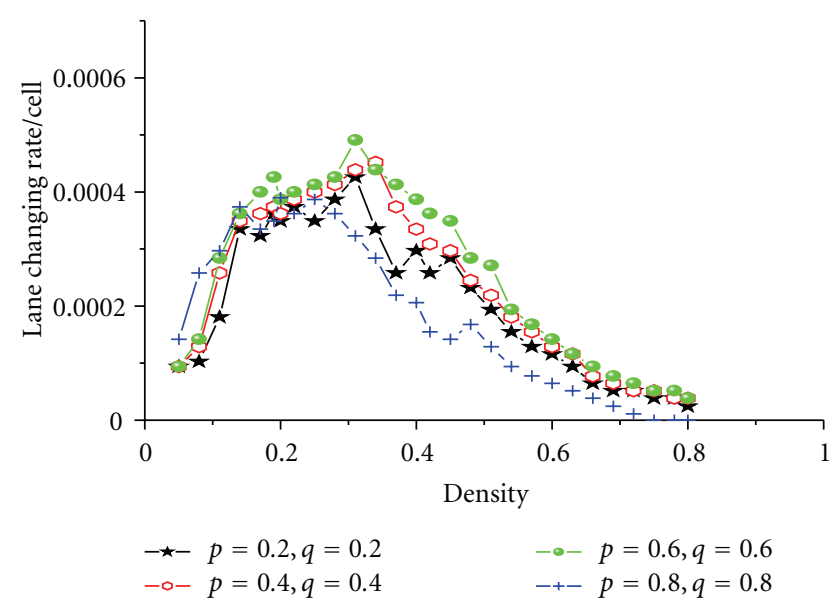

(c)

FIGURE 1: (a) Relationship between lane changing rate and density at braking probability $p=0.0$. (b) Relationship between lane changing rate and density at s-t-s probability $q=0.0$. (c) Relationship between lane changing rate and density at non zero values of braking probability $p$ and s-t-s probability $q$.

lane. With higher values of parameters $p$ and $q$, both criteria are fulfilled as the fluctuations of the distance between consecutive vehicles become larger. Figure 1(c) shows the lane-changing behavior of vehicles when both factors act together. With higher values of both parameters, safety criteria are not fulfilled and lane-changing rate again reduces drastically. Figure 2 describes the lane changing behavior of the Nasch model when simulated with implementation of s-t-s rule. Parameter $q$ has more effect than parameter $p$ on lane-changing behavior among vehicles. When both parameters are working together, the lane-changing tendency among vehicles increases. It can be seen that the results simulated from the Nasch model and modified cell model are close to each other except the magnitude of lane change rate/cell because of the reduced cell size.

\section{Single Lane versus Two Lanes}

A detailed comparison of the single-lane model with the corresponding two-lane model with effects of parameters $p$ and $q$ is shown in Figure 3. The graph shows rise in maximum flow $q_{\max }$, when simulating two-lane traffic as compared to one-lane system. This is due to the fact that some vehicles move into the other lane to avoid traffic jam and continuously contribute to flow. Now it is very interesting to observe how flow of the system is affected by the parameters $p$ and $q$ when acting alone. Since parameter $p$ affects all the vehicles with equal probability, whereas parameter $q$ affects only static vehicle that is, vehicles blocked by leading vehicles in the previous time step, the parameter $p$ is more responsible than parameter $q$ in reducing the throughput. However when values of $p$ and $q$ are high, this reduction can be significant. In the low-density region $\left(\rho<\rho_{c}\right)$, the average velocity of the traffic system is close to maximum velocity $V_{\max }$. Therefore there is not much difference in throughput of the system with nonzero $p$ as shown in Figure 3. But with zero value of parameter $p$ flow rises even in low-density region because parameter $q$ comes into action only in high density region $\left(\rho>\rho_{c}\right)$ as it affects only jammed vehicles. 




FIGURE 2: Relationship between lane-changing rate and density obtained from the Nasch model at various values of $p$ and $q$.

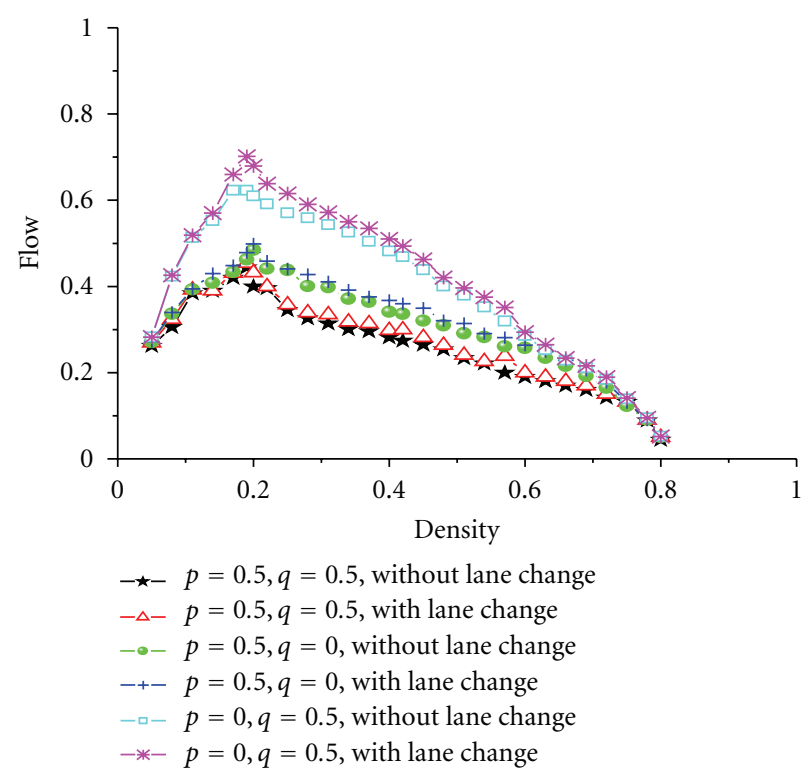

FIgURe 3: Relationship between density and flow with lane change and without lane change at various values of parameters $p$ and $q$.

Figure 4 describes the effect of parameters $p$ and $q$ over average velocity of the two-lane system with maximum speed $V_{\max }=60$ cells $/ \delta t$. In high-density region, average speed becomes the decreasing function of density. One can understand that average speed decreases linearly with the increase in the density of the system. But difference in speed variance is noticeable with implementation of nonzero parameters $p$ and $q$. During the laminar flow phase, speed variance is negligible but starts to increase with onset of maximum flow. In the low-density region, average speed is near to maximum speed when parameter $q$ acts alone and maximum speed variance is observed in maximum flow region. Thus with zero value of parameter $p$, the density of maximum flow is in the region of density of maximum

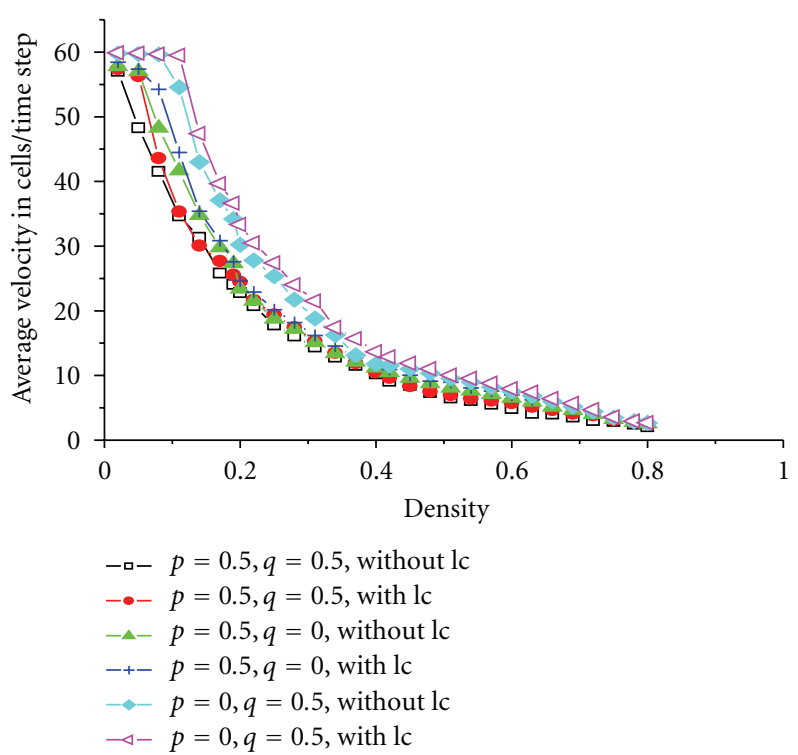

FIGURE 4: Relationship between density and average velocity with lane changing and without lane changing at various values of braking probability $p$ and s-t-s probability $q$.

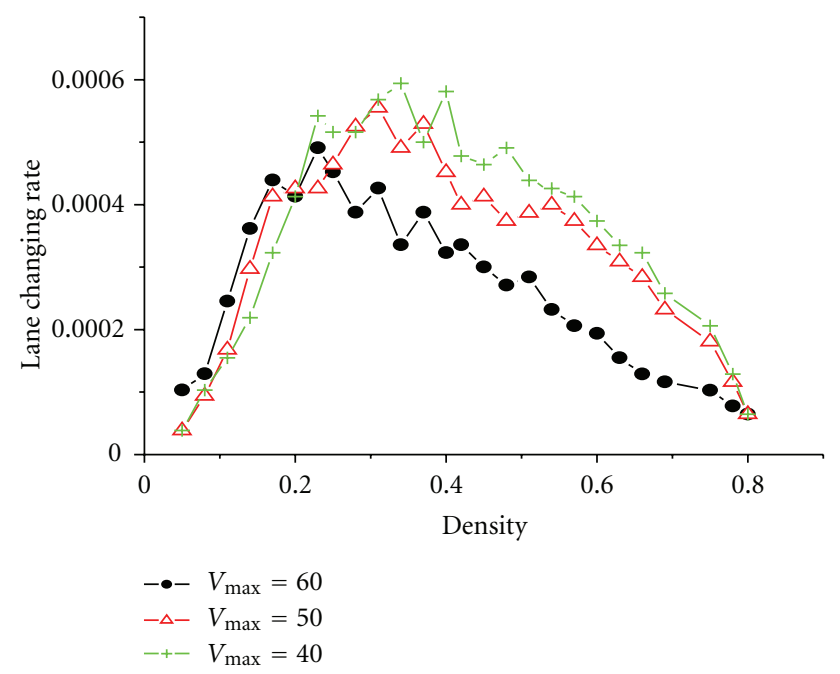

FIGURE 5: Relationship between density and lane-changing rate at various values of maximum velocity $V_{\max }$ with nonzero values of parameter $p$ and $q$.

speed variance. But this result is not true for nonzero value of parameter $p$. In this case speed variance is observed even in low-density region. This is because parameter $p$ comes into action long before the parameter $q$. Figure 5 shows the effect of maximum speed limit over lane-changing behavior of vehicles. In low-density region, increase in maximum speed limit $V_{\max }$ results in only limited amount of increase in lane-changing rate. But as density surpasses critical density $\left(\rho_{c} \sim 0.19\right)$, the lane-changing rate decreases with $V_{\max }$. This is due to the fact that in high-density region safety criteria are not fulfilled with higher values of $V_{\max }$, that is, the condition for looking back in target lane for safety point of view is not fulfilled. 


\section{Conclusion}

We have extended the $\mathrm{BJH}$ model to two-lane model with a reduced cell size and a variable acceleration rate. The reduced-cell-size CA model is more appropriate to describe the finer variability in traffic flow rather than the Nasch model with cell size $\delta x=7.5 \mathrm{~m}$. We investigated the effects of braking probability and s-t-s probability over lane-changing maneuver. We studied via simulation how vehicles fulfill both the incentive and safety criteria with higher values of braking and s-t-s probabilities. Combined effect of braking rule and s-t-s rule increases the effectiveness of the lane changes because gap acceptance is increased between the vehicles. We also compared the flow and average velocity of the two-lane system with single lane under the influence of braking probability and s-t-s probability. S-t-s probability has more effect than braking probability on lane-changing rate. It is also interestingly observed that the maximum lane changing frequency occurs long after the critical density $\rho_{c}$ of maximum throughput. Lane-changing frequency increases with introduction of nonzero parameters $p$ and $q$. This means that lane changing does little to increase throughput. Since more frequent lane changing means an increase in the likelihood of traffic accidents, the traffic should be operated at lower densities. In fact, the desired density should be smaller than both the density of maximum lane changing and maximum throughput. This will ensure traffic with few lane changes and with a small speed variance.

We also investigated the speed variance near the maximum flow and observed that density of maximum flow is in the region of density of maximum speed variance in case of nonzero values of braking probability. Thus presence of any amount of variability is sufficient to result in high speed variance. High speed variance means that different vehicles in the system have widely varying speeds. It means that a vehicle would experience frequent speed change per trip through the system. Since s-t-s rule in traffic flow enhances the lane-changing tendency among vehicles, combined effect of braking rule and s-t-s rule is significant in high-density region. Actually s-t-s rule reflects the feature of real driving and is distinct from general disorder rule. Therefore combined study of disorder rule with s-t-s rule is necessary for a safety point of view. Present model reveals all the features of two-lane traffic flow.

\section{Acknowledgment}

This work is partially supported by a grant-in-aid from the Council of Scientific and Industrial Research, India.

\section{References}

[1] K. Nagel and M. Schrekenberg, "A cellular automaton model for freeway traffic," Journal de Physique I, vol. 2, no. 12, pp. 2221-2229, 1992.

[2] M. Rickert, K. Nagel, M. Schreckenberg, and A. Latour, "Two lane traffic simulations using cellular automata," Physica A, vol. 231, no. 4, pp. 534-550, 1996.
[3] W. Knospe, L. Santen, A. Schadschneider, and M. Schrekenberg, "Disorder effects in cellular automata for two lane traffic," Physica A, vol. 265, no. 3-4, pp. 614-633, 1998.

[4] A. Awazu, "Dynamics of two equivalent lanes traffic flow model: self- organization of the slow lane and fast lane," Journal of Physical Society of Japan, vol. 64, no. 4, pp. 10711074, 1998.

[5] E. G. Campri and G. Levi, "A cellular automata model for highway traffic," The Europian Phyisical Journal B, vol. 17, no. 1, pp. 159-166, 2000.

[6] L. Wang, B. H. Wang, and B. Hu, "Cellular automaton traffic flow model between the Fukui-Ishibashi and NagelSchreckenberg models," Physical Review E, vol. 63, no. 5, Article ID 056117, 5 pages, 2001.

[7] W. Knospe, L. Santen, A. Schadschneider, and M. Schreckenberg, "A realistic two-lane traffic model for highway traffic," Journal of Physics A, vol. 35, no. 15, pp. 3369-3388, 2002.

[8] B. Jia, R. Jiang, Q. S. Wu, and M. B. Hu, "Honk effect in the two-lane cellular automaton model for traffic flow," Physica A, vol. 348, pp. 544-552, 2005.

[9] D. Chowdhury, L. Santen, and A. Schadschneider, "Statistical physics of vehicular traffic and some related systems," Physics Report, vol. 329, no. 4-6, pp. 199-329, 2000.

[10] R. J. Harris and R. B. Stinchcombe, "Ideal and disordered twolane traffic models," Physica A, vol. 354, no. 1-4, pp. 582-596, 2005.

[11] X. G. Li, B. Jia, Z. Y. Gao, and R. Jiang, "A realistic two-lane cellular automata traffic model considering aggressive lanechanging behavior of fast vehicle," Physica A, vol. 367, pp. 479486, 2006.

[12] H. B. Zhu, L. Lei, and S. Q. Dai, "Two-lane traffic simulations with a blockage induced by an accident car," Physica A, vol. 388, no. 14, pp. 2903-2910, 2009.

[13] C. Chen, J. Chen, and X. Guo, "Influences of overtaking on two-lane traffic with signals," Physica A, vol. 389, no. 1, pp. 141-148, 2010.

[14] C. Mallikarjuna and K. Rao, "Identification of a suitable cellular automata model for mixed traffic," Journal of the Eastern Asia Society for Transportation Studies, vol. 7, pp. 24542468, 2007.

[15] S. C. Benjamin, N. F. Johnson, and P. M. Hui, "Cellular automata models of traffic flow along a highway containing a junction," Journal of Physics A, vol. 29, no. 12, pp. 3119-3127, 1996.

[16] A. Clarridge and K. Salomaa, "Analysis of a cellular automaton model for car traffic with a slow-to-stop rule," Theoretical Computer Science, vol. 411, no. 38-39, pp. 3507-3515, 2010. 



Submit your manuscripts at

http://www.hindawi.com
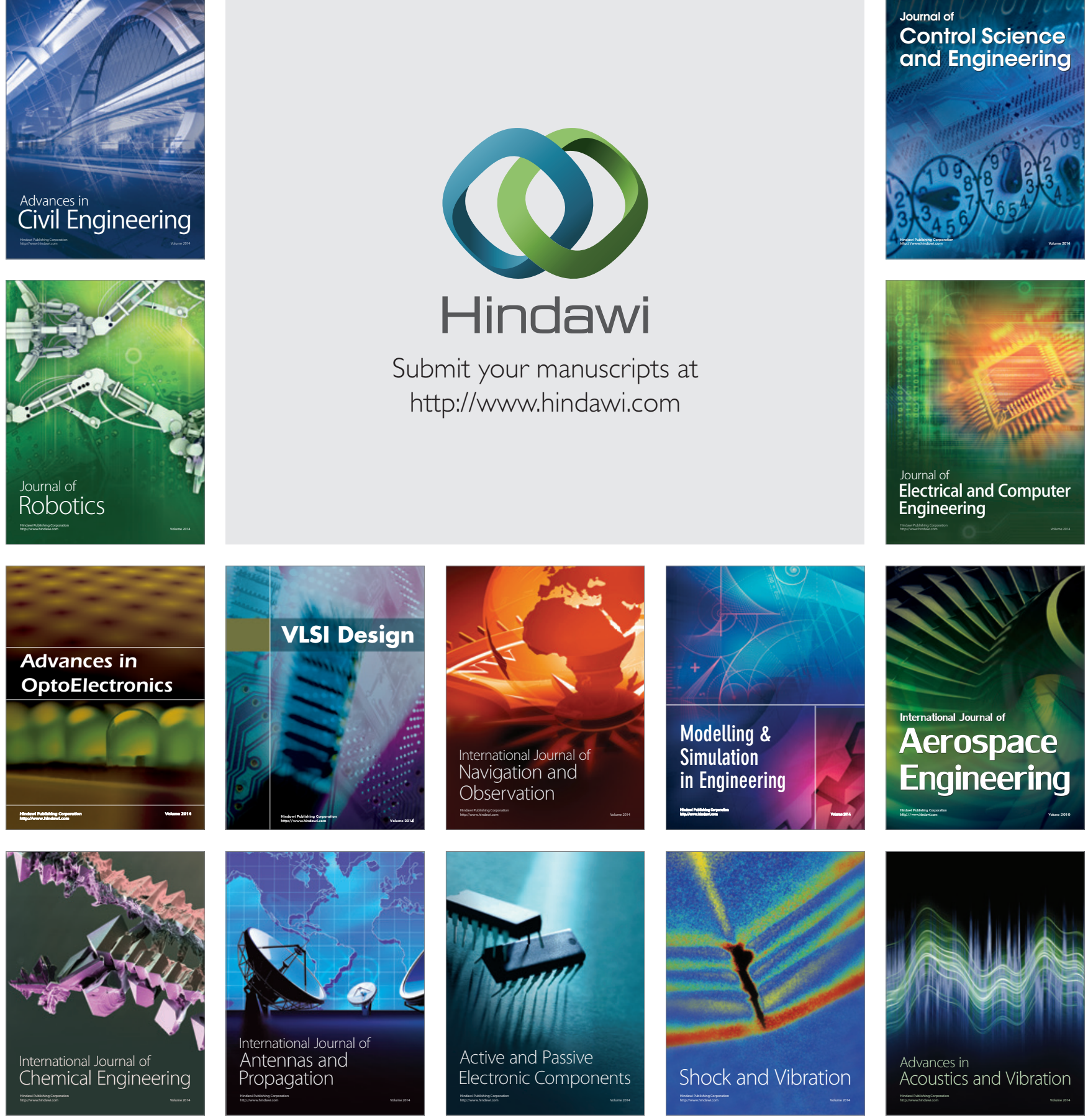